\title{
Influence of Solvent Quality on the Self-Organization of Archetypical Hairy Rods-Branched and Linear Side Chain Polyfluorenes: Rodlike Chains versus "Beta-Sheets" in Solution
}

\author{
M. Knaapila, $*, \dagger$ V. M. Garamus,${ }^{\ddagger}$ F. B. Dias,${ }^{\dagger}$ L. Almásy, ${ }^{\S}$ F. Galbrecht,,$\|$ A. Charas,${ }^{\perp}$ \\ J. Morgado, ${ }^{\perp}$ H. D. Burrows, ${ }^{\#}$ U. Scherf,,$"$ and A. P. Monkman ${ }^{\dagger}$ \\ Department of Physics, University of Durham, South Road, Durham DH1 3LE, UK; GKSS Research \\ Centre, Max-Planck-Strasse 1, D-21502 Geesthacht, Germany; Research Institute for Solid State \\ Physics and Optics, P.O. Box 49, Budapest-1525, Hungary; Macromolekulare Chemie, Institut für \\ Polymertechnologie, Bergische Universität Wuppertal, Gauss-Strasse 20, D-42097 Wuppertal, \\ Germany; Instituto de Telecomunicações, Instituto Superior Técnico, Av. Rovisco Pais, P-1049-001 \\ Lisboa, Portugal; and Departamento de Química, Universidade de Coimbra, P- 3004-535 Coimbra, \\ Portugal
}

Received April 20, 2006; Revised Manuscript Received June 26, 2006

\begin{abstract}
We report on the nanoscale structure and solvent-induced phase behavior of two, nearly similar $\pi$-conjugated hairy-rod polymers, branched side chain poly[9,9-bis(2-ethylhexyl)fluorene-2,7-diyl] (PF2/6) and linear side chain poly[9,9-dioctylfluorene-2,7-diyl] (PFO or PF8), in good and bad (or poor) solvents-deuterated toluene and deuterated methylcyclohexane $(\mathrm{MCH})-$ at $20^{\circ} \mathrm{C}$. Small-angle neutron scattering (SANS) measurements exploiting contrast variation with side chain deuterated PFO polyfluorene have been employed and complemented by optical absorption measurements. In toluene both PF2/6 and PFO adopt an elongated (rodlike) conformation containing predominantly only a single polymer chain (diameter of the order of $1 \mathrm{~nm}$ ), which indicates dissolution down to the molecular level. In contrast, in $\mathrm{MCH}, \mathrm{PF} 2 / 6$ shows an elongated structure while PFO forms sheetlike structures (characteristic thickness of $2-3 \mathrm{~nm}$ ), thus dissolving down to the "colloidal" level. The elongated structure of PFO consists of individual polymer chains adopting dominantly a conformational isomer $\mathrm{C}_{\alpha}$. The thickness of sheetlike PFO particles corresponds to that of around two polymer layers and side chain contrast variation gives an evidence for an even distribution of the backbones within the sheets. These sheets are potentially an initial stage of PFO crystallization and also contain conformational isomer $\mathrm{C}_{\beta}$ of those chains observed in the so-called beta-phase (or beta-sheets) in the solid state. The observed phenomena were not found to depend on concentration over the concentration range $5-10 \mathrm{mg} / \mathrm{mL}$.
\end{abstract}

\section{Introduction}

Understanding of macromolecular self-organization ${ }^{1}$ is essential in the physics and materials science of hairy-rod polymers. $^{2}$ An important class of self-assembling hairy rods are $\pi$-conjugated polymers ${ }^{3}$ such as poly(fluorene-2,7-diyl)s $(\mathrm{PFs})^{4-6}$ with excellent stability and optoelectronic versatility. The branched side chain poly[9,9-bis(2-ethylhexyl)fluorene-2,7-diyl] (PF2/6),${ }^{7-13}$ corresponding oligomers, ${ }^{14,15}$ and linear side chain poly[9,9-dioctylfluorene-2,7-diyl] (PFO or PF8), ${ }^{16-27}$ and its homologues such as poly[9,9-dihexylfluorene-2,7-diyl] $(\mathrm{PF} 6)^{28}$ represent structural archetypes of PFs. These polymers are excellent model compounds and are heavily studied in the solid state and films. Distinctive to all the findings to date is the striking physical difference between these two materials despite their close chemical resemblance; they have almost the same chemical formula and chemical structure (Figure 1). Yet the major distinguishing feature is that of the chain morphology. PF2/6 always adopts a helix with a single ensemble average distribution in chain conformations leading to nematic, hexagonal, and isotropic phases. Consequently, the photoabsorption and photoluminescence of PF2/6 are essentially independent of

\footnotetext{
University of Durham.

$¥$ GKSS Research Centre.

$\S$ Research Institute for Solid State Physics and Optics.

"Bergische Universität Wuppertal.

${ }^{\perp}$ Instituto de Telecomunicações.

\# Universidade de Coimbra.

* Corresponding author: Tel +44-191-33-43558, Fax +44-191-3343585, e-mail matti.knaapila@durham.ac.uk.
}

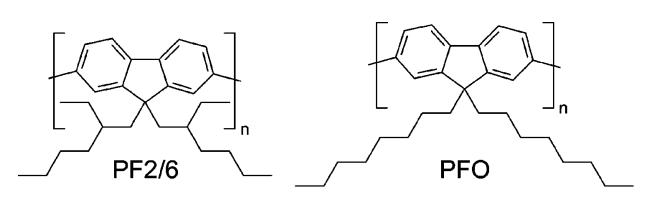

Figure 1. Chemical structures of poly[9,9-bis(2-ethylhexyl)fluorene2,7-diyl] (PF2/6) and poly[9,9-dioctylfluorene-2,7-diyl] (PFO).

its phase and processing. In contrast, the chain planarity of PFO can vary in wide limits representing various different conformational isomers (denoted as $\mathrm{C}_{\alpha}, \mathrm{C}_{\beta}, \ldots$ ) defined by the torsional angle between the monomers. ${ }^{26}$ This variation is in turn reflected by the polymorphism of crystalline $\alpha$-phase and metastable $\alpha^{\prime}$ phase, mesomorphic and metastable $\beta$-phase, and amorphous, glassy, nematic, and isotropic phases with complex transitions. Accordingly, the isomers and different "phases" of PFO show significant photophysical differences despite their identical chemical structure.

Not surprisingly, most efforts in structural and phase behavioral investigations of PF2/6 and PFO have hitherto been focused on the studies of thin films, the application environment of $\pi$-conjugated polymers. Instead, the literature of solution structures of these polymers ${ }^{12,29,30}$ is less comprehensive. However, as shown for example for poly $(9,9$-dioctylfluoreneco-benzothiadiazole), ${ }^{31}$ thin film morphology is related to the solution structure and can be controlled by varying the casting solvent. A prime parameter to control the solution structure of polymers is of course the phenomenological solvent quality: Here a solvent where the polymer chain maximizes the 
polymer-solvent contacts becoming extended is referred to as a good solvent. Correspondingly, a solvent where the flexible polymer minimizes solvent-polymer contacts to such a degree where the polymer chain forms a condensed globule is referred as a poor solvent. ${ }^{1}$ Rigid $^{32}$ or semiflexible ${ }^{33}$ polymers are not able to form close collapsed globules, and therefore the term poor solvent refers rather to the phenomenological state where several chains start to form condensed aggregates. When limiting our discussion to $\pi$-conjugated hairy-rod polymers, we may for example note that poly( $p$-phenylenesulfonate $)$ s form cylindrical ${ }^{34}$ and poly[2-methoxy-5-(2'-ethylhexyloxy)-1,4-phenylenevinylene] (MEH-PPV) disklike ${ }^{35}$ micelles in a poor solvent.

PFO is known to show solvatochromic effects. ${ }^{36}$ In one particular example it has been demonstrated that the variation between a phenomenologically good (toluene) and a poor solvent (methylcyclohexane (MCH)) influences absorption/ emission of PFO. ${ }^{37}$ This effect depends on temperature but is independent of the polymer concentration in the range of 3-25 $\mu \mathrm{g} / \mathrm{mL} .{ }^{37}$ On the basis of optical observations, the following hypothesis was put forward. It has been thought that PFO forms "ordered" regions which act as efficient energy traps and quench the fluorescence from the "disordered" regions. As it is controversial how order is realized in solution, explicit structural data would be useful.

In the above mentioned previous study the "ordered" regions of PFO were denoted as a $\beta$-phase. ${ }^{37}$ Attention should be paid to the loose variations in the interpretation and/or terminology concerning the $\beta$-phase. This may either refer to the intermolecular solid-state structure or one conformational intramolecular isomer with torsion angle of the order of $160^{\circ}-165^{\circ}$. Most generically, the term $\beta$-phase simply refers to the low-energy absorption/emission state of the chain, rigorously disregarding any implications about the 3-dimensional order. All this may also be a phenomenological oversimplification, and the origin of the discussed absoption/emission state may stem from far more complicated self-organization in terms of mean packing between two distinct average chain shapes over short distances. In general, it is inaccurate or wrong to talk about solution using terms that elsewhere refer to the real solid-state order, and therefore the analogy is phenomenological at best. The explicit structural studies of the solutions where the $\beta$-phase is suggested to exist might presumably clarify this issue in part.

Small-angle X-ray scattering (SAXS $)^{38,39}$ and small-angle neutron scattering (SANS) $)^{40-43,35}$ are commonly used to study $\pi$-conjugated hairy-rod polymers in solutions. The characteristics of $\mathrm{PF} 2 / 6^{29,12}$ and $\mathrm{PF}$ copolymers ${ }^{44}$ have been probed in deuterated toluene, but studies in other solvents seem to be scarce. As small-angle scattering probes specifically the nanometer scale, the particular dimension of self-organized phases, this is an ideal but little utilized tool.

In this paper, we present a SANS study of PF2/6 and PFO in toluene and $\mathrm{MCH}$ at $20{ }^{\circ} \mathrm{C}$ with contrast variation achieved using deuterated side chain PFO. The same systems were also studied by optical density measurements. The objectives of this paper are threefold. First, the aim is to demonstrate essential differences in the solution dependent phase behavior of the archetypical hairy-rod polyfluorenes in good and poor solvents. Second, the aim is to provide a quantitative structural characterization for the prime PFs in the concentration range used in film formation in optoelectronic applications. The particular aim is to extend our previous experimental and modeling studies of PF2/6 in toluene. ${ }^{12}$ Third, the aim is to relate the findings of the previous optical study of $\mathrm{PFO}^{37}$ in structural features, in particular in the case where absorption and emission data suggest the existence of $\beta$-phase in solution. Considering the significant difference in concentration between these two studies, any analogy is as yet quite phenomenological.

We build our arguments on the decays of the SANS curves with the magnitude of the scattering vector and thereafter on the indirect Fourier transform method and on the fits to the form factor of rod and sheet as well as characteristic features found in the optical densities. We find that both PF2/6 and PFO assume a thin $(\sim 10 \AA)$ and long (>700 $\AA$ ) rodlike r $^{45}$ conformation in toluene. However, the situation is different in $\mathrm{MCH}$, where $\mathrm{PF} 2 / 6$ adopts an elongated structure, whereas PFO forms large $(\sim 1000 \AA)$ and thin $(\sim 20 \AA)$ sheetlike aggregates. PFO takes predominantly a conformation isomer $\mathrm{C}_{\alpha}$ in toluene and a combination of at least $\mathrm{C}_{\alpha}$ and $\mathrm{C}_{\beta}$ in $\mathrm{MCH}$. The thicknesses of all rodlike objects are in accord with those of single polymer chains. The thickness of PFO sheets in $\mathrm{MCH}$ corresponds in turn to the thickness of around 2-3 polymer layers. The internal structure of these aggregates cannot be revealed in detail, but the backbones are potentially evenly distributed within the sheets. Thus, the rodlike particles indicate dissolution down to molecular and the sheetlike particles down to colloidal level. No evidence for three-dimensional aggregates (at or beyond nanoscale) was seen. The observed phenomena were found to be essentially concentration independent over the concentration range $5-10 \mathrm{mg} / \mathrm{mL}$.

\section{Experimental Section}

PF2/6 $\left(M_{\mathrm{n}}=39 \mathrm{~kg} / \mathrm{mol}\right.$, the weight-averaged molecular weight $\left.\left(M_{\mathrm{w}}\right)=58 \mathrm{~kg} / \mathrm{mol}\right)$ and PFO $\left(M_{\mathrm{n}}=49 \mathrm{~kg} / \mathrm{mol}, M_{\mathrm{w}}=132 \mathrm{~kg} / \mathrm{mol}\right)$ (Figure 1) were prepared following the microwave-assisted Yamamoto-type polymerization with $\mathrm{Ni}(\mathrm{COD})_{2}$ catalyst. ${ }^{6,46}$ Poly[9,9-bis$\left(\right.$ di- $n$-octyl- $\left.d_{17}\right)$ fluorene-2,7-diyl] (PFO- $\left.d_{34}\right)\left(M_{\mathrm{n}}=119 \mathrm{~kg} / \mathrm{mol}, M_{\mathrm{w}}\right.$ $=194 \mathrm{~kg} / \mathrm{mol}$ ) was prepared similarly but with the nondeuterated 2,7-dibromo-9,9-dioctylfluorene monomer being replaced by the deuterated equivalent containing octyl- $d_{17}$ side chains. The estimated densities used were $0.97 \mathrm{~g} / \mathrm{cm}^{3}$ for PF $2 / 6^{7}$ and $1.0 \mathrm{~g} / \mathrm{cm}^{3}$ for PFO. ${ }^{23}$

For SANS, the polymers were dissolved either in toluene- $d_{8}$ (99.5\% D, Cambridge Isotope Laboratories Inc.) or in methylcyclohexane- $d_{14}(99.6 \%$ D, GOSS Scientific Instruments Ltd.), MCH$d_{14}$. Concentrations were $5-10 \mathrm{mg} / \mathrm{mL}$. PFO and PFO- $d_{34}$ were dissolved in toluene and PF2/6 in both solvents by stirring for 5 min at $20{ }^{\circ} \mathrm{C}$. These samples were then measured as such. PFO is not soluble in $\mathrm{MCH}$ at room temperature at the highest concentrations. Therefore, it was first heated to $80-85^{\circ} \mathrm{C}$ and stirred for 5 min until completely transparent solutions were observed. These samples were then cooled from $80-85^{\circ} \mathrm{C}$ to $-25{ }^{\circ} \mathrm{C}$ for $30 \mathrm{~min}$ and then slowly warmed to $20^{\circ} \mathrm{C}$ before measurements. Parallel sets of materials were cooled to $20^{\circ} \mathrm{C}$ without the low-temperature cycle. Prepared MCH- $d_{14}$ solutions of PFO are very viscous or gellike at room temperature, and PFOs tend to macrophase separate in a few days after preparation. However, all measurements were done immediately after sample preparation, and macrophase separation was not observed during the measurements (in a few hours).

SANS measurements were performed using the Yellow Submarine instrument at the BNC in Budapest, Hungary, ${ }^{47}$ and the SANS-1 instrument at the GKSS Research Centre in Geesthacht, Germany. ${ }^{48}$ Several sample-to-detector distances (from 0.7 to $9.7 \mathrm{~m}$ ) and wavelength from 5 to $12 \AA$ were employed to cover the range of scattering vector $q$ from 0.005 to $0.3 \AA^{-1}$. The samples were filled in Hellma quartz cells of $5 \mathrm{~mm}$ path length and placed in a thermostated holder, kept at $20.0 \pm 0.5^{\circ} \mathrm{C}$. The raw scattering patterns were corrected for sample transmission, room background, and sample cell scattering by conventional procedures. ${ }^{49}$ The isotropic $2 \mathrm{D}$ scattering patterns were azimuthally averaged, converted to an absolute scale, and corrected for detector efficiency dividing by the incoherent scattering spectra of $1 \mathrm{~mm}$ thick pure water. The scattering from deuterated solvents used for the sample preparation was subtracted as a background; the small incoherent 
scattering due to the nondeuterated polymer was disregarded. The data for each sample was collected for $6 \mathrm{~h}$ on average. Overall, the SANS experiments were reproduced using several parallel samples and two distinct instruments.

The SANS patterns absolute intensities were analyzed using models of rodlike and disklike (or sheetlike) objects according to Pedersen ${ }^{50-52}$ and using a model independent indirect Fourier transform method (IFT) developed by Glatter. ${ }^{53}$

For a solution of stiff cylinders the general expression of the scattering intensity is written as

$$
\frac{\mathrm{d} \Sigma(q)}{\mathrm{d} \Omega} \frac{1}{c}=\frac{1}{M} P(q) S^{\prime}(q)
$$

where $M$ is the mass of particle and $P(q)$ is the scattering of a single particle. It reflects scattering contrast and depends on the length and radius of the homogeneous cylinder, $L$ and $R . S^{\prime}(q)$ is the effective structure factor. It reflects the interaction among particles and depends on volume fraction, $L$ and $R$.

For sheetlike particles, the general expression of scattering intensity is written as

$$
\frac{\mathrm{d} \Sigma(q)}{\mathrm{d} \Omega} \frac{1}{c} \sim \frac{\sin (T q)}{T q} \int \frac{2}{q^{2} R^{2}}\left[1-\frac{J_{1}(2 q R)}{q R}\right] f(r) \mathrm{d} r
$$

The polydispersity of the radius $R$ can be accounted by the means of the Schultz distribution $f(R)$ as

$$
f(R)=\left[\frac{p+1}{\langle R\rangle}\right]^{p+1}\left[\frac{R^{p}}{\Gamma(p+1)}\right] \exp \left[-\frac{(p+1) R}{\langle R\rangle}\right]
$$

where $\langle R\rangle$ is the average sheet radius and $p$ is the polydispersity index. The latter is related to the spread of the radius distribution as $\sigma_{\mathrm{R}}=\Delta R /\langle R\rangle=(p+1)^{-1 / 2}$.

In the IFT analysis, the scattering intensities of rodlike particles are related to the pair distance distribution function of cross section, $\tilde{p}_{\mathrm{CS}}(r)$, as

$$
\frac{\mathrm{d} \Sigma(q)}{\mathrm{d} \Omega} \frac{1}{c}=\left(\frac{\pi}{q}\right) 2 \pi \int_{0}^{\infty} \tilde{p}_{\mathrm{CS}}(r) J_{0}(q r) r \mathrm{~d} r=\left(\frac{\pi}{q}\right) I_{\mathrm{CS}}(q)
$$

where $J_{0}$ is the zeroth-order Bessel function and $I_{\mathrm{CS}}(q)$ is the crosssectional scattering intensity. For sheetlike particles the scattering intensity is related to the pair distance distribution function of thickness, $\tilde{p}_{\mathrm{T}}(r)$, as

$$
\frac{\mathrm{d} \Sigma(q)}{\mathrm{d} \Omega} \frac{1}{c}=\left(\frac{2 \pi}{q^{2}}\right) \pi \int_{0}^{\infty} \tilde{p}_{\mathrm{T}}(r) \cos (q r) \mathrm{d} r=\left(\frac{2 \pi}{q^{2}}\right) I_{\mathrm{T}}(q)
$$

where $I_{\mathrm{T}}(q)$ is the thickness scattering function.

From these expressions (eqs 1-5) the structural parameters of rodlike and sheetlike particles were calculated. More details of the procedures used are described in the Supporting Information.

Photoabsorption measurements of solutions were performed using a Perkin-Elmer Lambda 19 spectrometer and quartz cell of $10 \mu \mathrm{m}$ path length at $20 \pm 2{ }^{\circ} \mathrm{C}$. The small path length was necessary because of the high absorbance of the samples. The monochromator resolution was $1 \mathrm{~nm}$.

Differential scanning calorimetry (DSC) measurements were performed using a Perkin-Elmer Pyris 1 DSC. Dry samples of 2 $\mathrm{mg}$ and a sweep rate of $10^{\circ} \mathrm{C} / \mathrm{min}$ were used.

\section{Results}

Poly[9,9-bis(2-ethylhexyl)fluorene-2,7-diyl], PF2/6. PF2/6 has been previously studied using SANS in toluene solution. ${ }^{12,29}$ In toluene, PF2/6 has been found to form one-dimensional elongated particles over the concentration range $1-11 \mathrm{mg} / \mathrm{mL}$. These particles have been interpreted to be composed by individual chains, which imply that PF2/6 is dissolved down to the molecular level. Similar results have been qualitatively

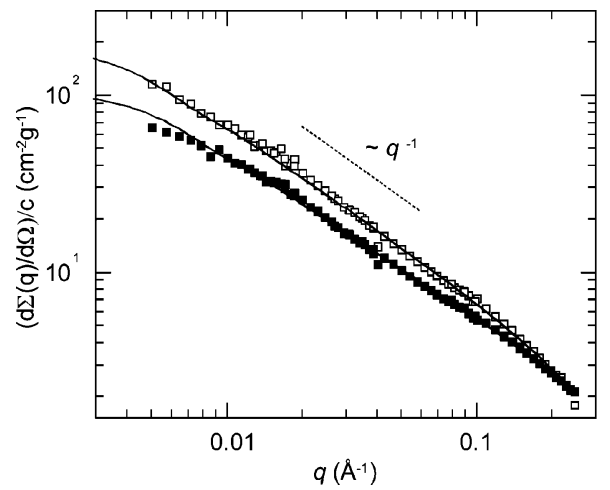

Figure 2. SANS data of PF2/6 in toluene- $d_{8}$ (solid squares) and in $\mathrm{MCH}-d_{14}$ (open squares). Solid lines are the fits based on the cylindrical model. The slopes of the linear fits (not shown) are in turn, respectively, $0.96 \pm 0.01$ and $1.07 \pm 0.01$ in the interval $0.011-0.04 \AA^{-1}$ Concentration was $10 \mathrm{mg} / \mathrm{mL}$.

observed for polyfluorene-fluorenone random copolymers. ${ }^{44}$ In particular, the SANS curve of PF2/6 $\left(M_{\mathrm{n}}=91 \mathrm{~kg} / \mathrm{mol} M_{\mathrm{w}}\right.$ $=190 \mathrm{~kg} / \mathrm{mol})$ in toluene $(9 \mathrm{mg} / \mathrm{mL})$ has been found to be in accord with the molecular mechanics model of the 5/2-helical 20 -mer incorporated into the featureless toluene matrix, ${ }^{12}$ thus connecting experimental data to the reasonably sophisticated molecular model. The prior study ${ }^{12}$ also suggests that there is no concentration effect in the concentration regime used in the present work.

Figure 2 plots the SANS data of PF2/6 in $10 \mathrm{mg} / \mathrm{mL}$ toluene$d_{8}$ and $\mathrm{MCH}-d_{14}$ solutions. The shapes of curves are quite similar for both solvents. The difference in absolute values is due to different scattering contrast normalized to the density of the polymer in toluene- $d_{8}$ and $\mathrm{MCH}-d_{14}, \Delta \rho_{\mathrm{m}}$ (PFO in toluene- $\left.d_{8}\right)$ $=-5.2 \times 10^{10} \mathrm{~cm} / \mathrm{g}$ and $\Delta \rho_{\mathrm{m}}\left(\mathrm{PFO}\right.$ in MCH- $\left.d_{14}\right)=-6.2 \times$ $10^{10} \mathrm{~cm} / \mathrm{g}$. Unsurprisingly, the data of toluene- $d_{8}$ solution (Figure 2) are consistent with the previous findings ${ }^{12,29}$ with a slope of around -1 over large $q$ range, indicating one-dimensional rodlike structures. The slope obtained from the data of PF2/6 in $\mathrm{MCH}-d_{14}$ is also around -1 , suggesting essentially similar one-dimensional rodlike structures. SANS data were then analyzed further using two distinct approaches.

First, the data over the whole $q$ range were fitted to the model of stiff cylinders of length $L$ and radius $R$ taking excludedvolume interaction between particles into account. Solid lines in Figure 2 represent fits to the cylindrical (rodlike) model. Model fitting indicates very long and very thin particles in both cases, similar to our previous results on PF2/6/toluene solutions. ${ }^{12}$ A corresponding Holtzer plot representation without any sign of flexibility for Kuhn lengths of $4-20 \mathrm{~nm}$ is shown in the Supporting Information.

Second, the data in the large $q$ part $\left(q>0.02 \AA^{-1}\right)$ were analyzed using the IFT method assuming rodlike geometry. The parameters obtained are integral characteristics of cross-sectional radius of gyration, $R_{\mathrm{CS}, \mathrm{g}}$, and the cross-sectional scattering at zero angle, $I(0)_{\mathrm{CS}}$. From these parameters we can calculate an average radius of cross section in homogeneous approximation, $R_{\mathrm{c}}$, and the mass per unit length of rodlike object, $M_{\mathrm{L}}$ (see the Supporting Information).

Furthermore, the IFT method enables us to calculate the crosssectional pair distance distribution (correlation) function, $p(r)$. Figure 3 plots the cross-sectional pair distance distribution functions corresponding to the data shown in Figure 2. It is conspicuous that the diameter of cross section of PF $2 / 6$ is smaller in $\mathrm{MCH}-d_{14}(8 \AA)$ than in toluene- $d_{8}(12 \AA)$. This indicates that toluene swells the polymer presumably by allowing the side chains to elongate. 


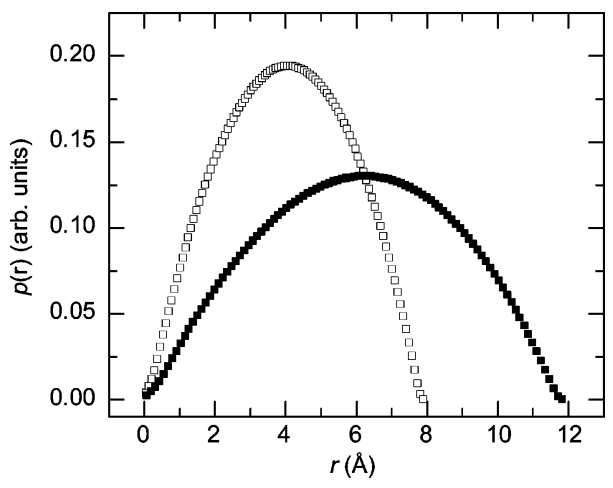

Figure 3. Cross-sectional pair distance distribution functions, $p(r)$, obtained from the SANS data of PF2/6 in toluene- $d_{8}$ (solid squares) and in $\mathrm{MCH}-d_{14}$ (open squares).

The visual consideration of Figure 2 shows that the tail of the curve of the MCH- $d_{14}$ solution falls more steeply than that of toluene- $d_{8}$. Therefore, one might expect that the radius of $\mathrm{PF} 2 / 6$ is larger in the former solution while the analysis shows the opposite. This intuitive disagreement stems from the fact that the incoherent residual background relative to the real scattering is higher in toluene- $d_{8}$ than in $\mathrm{MCH}-d_{14}$. This effect is distinctive here as the particles are very thin, and therefore we need to probe them at very large $q$. However, the full form factor together with the incoherent background was taken into the account in the IFT analysis and model fitting.

Essential structural parameters fitted to these data both by modeling of homogeneous cylinders and IFT are compiled in Table 1. The difference in radius of gyration of cross section obtained by IFT and model fitting (the former is larger) suggests that the scattering length density is higher in the outer shell of the cross section. We note that the obtained lengths of cylinders are actually near the limit of our instrument $\left(\pi / q_{\min } \approx 1000 \AA\right)$ and should be considered as an approximate lower limit. On the whole, Table 1 indicates strongly that PF2/6 maintains its rodlike conformation both in toluene- $d_{8}$ and $\mathrm{MCH}-d_{14}$, becoming somewhat thinner in $\mathrm{MCH}-d_{14}$.

The results also indicate that the mass per unit length of PF2/6 is essentially the same, $5.5 \times 10^{-15} \mathrm{~g} / \mathrm{cm}$, in toluene- $d_{8}$ and in $\mathrm{MCH}-d_{14}$ (Table 1). The mass per unit length of PF2/6 was also calculated from the chemical formula and standard solidstate molecular parameters ${ }^{54}$ taking a hexagonal lattice with lattice parameters $a=b=16.7 \AA$ and $c=40.4 \AA$ (a length of five monomers) and with density $\approx 0.97 \mathrm{~g} / \mathrm{cm}^{3} .{ }^{37}$ The result is the same order of magnitude as the measured one, $8 \times 10^{-15}$ $\mathrm{g} / \mathrm{cm}$.

Poly[9,9-dioctylfluorene-2,7-diyl], PFO. Solvent-Induced Self-Organization of PFO. Figure 4 plots SANS data of $10 \mathrm{mg} /$ $\mathrm{mL}$ PFO in toluene- $d_{8}$ and in MCH- $d_{14}$. It is conspicuous that the change of solvent leads to the drastic changes in scattering intensity. At $q=0.01 \AA^{-1}$ this is 100 times higher for the PFO in $\mathrm{MCH}-d_{14}$ than for PFO in toluene- $d_{8}$. Furthermore, the decay of scattering intensity changes distinctively from -1 (toluene$\left.d_{8}\right)$ to $-2\left(\mathrm{MCH}-d_{14}\right)$. The corresponding Holtzer plot of PFO in toluene- $d_{8}$ (Supporting Information) indicates rigidity in the length scale $2-20 \mathrm{~nm}$. These observations indicate significant

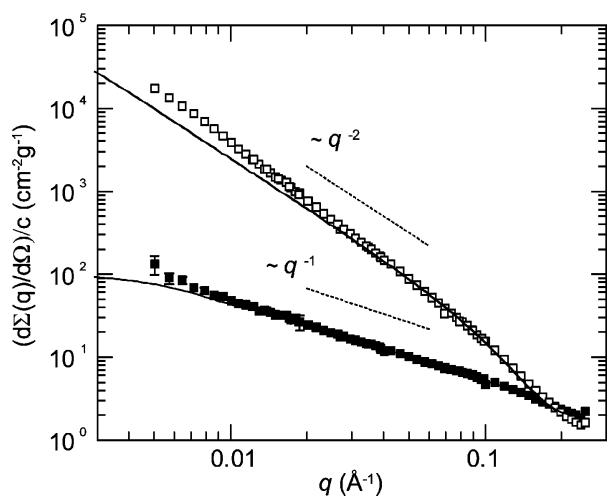

Figure 4. SANS data of PFO in toluene- $d_{8}$ with concentration of 10 $\mathrm{mg} / \mathrm{mL}$ (solid squares) and in $\mathrm{MCH}-d_{14}$ (open squares). Solid lines are the fits of the data based on the models of rodlike and disklike objects. Dashed lines show the -1 and -2 decays for comparison.

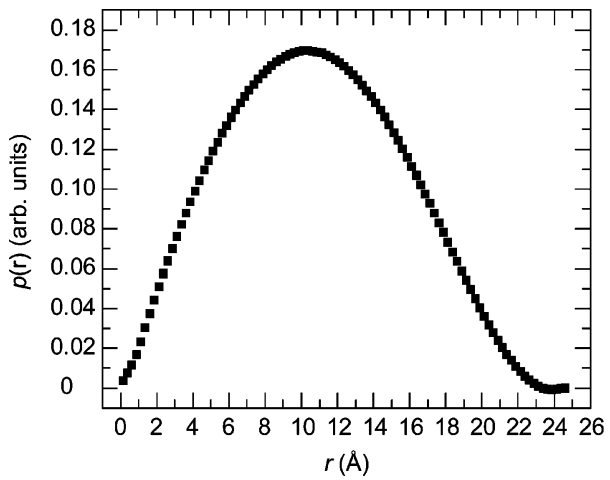

Figure 5. Pair distance distribution function obtained from the SANS data of PFO in toluene- $d_{8}$ shown in Figure 4.

structural reorganization of PFO when the solvent is changed from toluene- $d_{8}$ to $\mathrm{MCH}-d_{14}$. In toluene- $d_{8}$ there are rodlike particles similar to those in PF2/6 solutions, but in $\mathrm{MCH}-d_{14}$ there are much larger objects with apparent planar geometry (sheets or disks). We first discuss the rodlike PFO particles in toluene- $d_{8}$. Then we move on the sheetlike PFO aggregates in $\mathrm{MCH}-d_{14}$.

Rodlike PFO Particles in Toluene. The data analysis in the case of PFO/toluene- $d_{8}$ was identical to that performed for PF2/6 solutions, and the results are presented in Figure 4, where a solid line represents fit to the cylindrical model. Figure 5 plots the corresponding pair distribution function obtained by IFT. For comparison, Figure 6 plots SANS data of lower concentration $(5 \mathrm{mg} / \mathrm{mL})$ PFO in toluene- $d_{8}$ alongside the higher one $(10$ $\mathrm{mg} / \mathrm{mL}$ ) normalized to the concentration. Solid lines are the fits to the model of stiff cylinders. Deviations between the data could indicate interaction between PFO particles in toluene- $d_{8}$ under the conditions studied. However, the data for both concentrations are practically identical, suggesting that there is no major aggregation of polymer molecules in these concentrations. This implies that the interaction between polymers is quite low. Essential structural parameters obtained from both methods are listed in Table 2.

Table 1. Parameters Fitted to the SANS Data of $10 \mathrm{mg} / \mathrm{mL}$ PF2/6 in Toluene- $d_{8}$ and in MCH- $d_{14}{ }^{a}$

\begin{tabular}{cccccc}
\hline material & $L(\AA)$ & $R(\AA)$ & $R_{\mathrm{CS}, \mathrm{g}}(\AA)$ & $R_{\mathrm{c}}(\AA)$ & $M_{\mathrm{L}}(\mathrm{g} / \mathrm{cm}) \times 10^{-15}$ \\
\hline $\mathrm{PF} 2 / 6$ in toluene- $d_{8}$ & $740 \pm 80$ & $5.4 \pm 0.2$ & $4.7 \pm 0.1$ & $6.6 \pm 0.2$ & 5.47 \\
PF2/6 in MCH- $d_{14}$ & $920 \pm 90$ & $3.3 \pm 0.1$ & $3.1 \pm 0.1$ & $4.4 \pm 0.2$ & 5.54
\end{tabular}

${ }^{a} L$ and $R$ respectively are the length and radius of homogeneous cylinder obtained by the model fitting. $R_{\mathrm{CS}, \mathrm{g}}$ and $R_{\mathrm{c}}$ are respectively the cross-sectional radius of gyration and the radius and corresponding radius of homogeneous cross section. $M_{\mathrm{L}}$ is the measured mass per unit length obtained by the IFT method. 


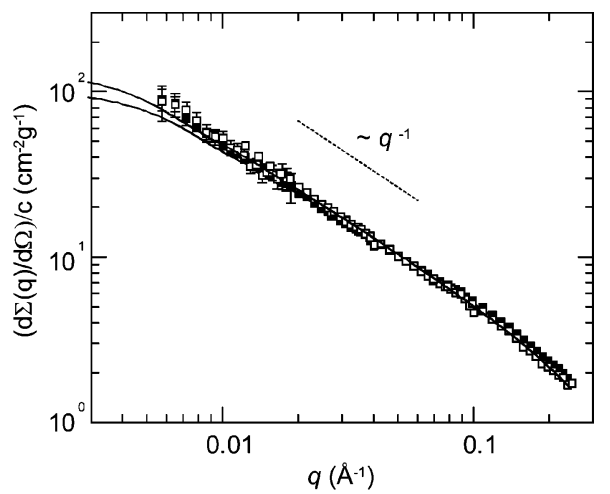

Figure 6. SANS data PFO in $5 \mathrm{mg} / \mathrm{mL}$ (open squares) and $10 \mathrm{mg} / \mathrm{mL}$ (solid squares) toluene- $d_{8}$ normalized to the concentration. Solid lines are fits to model of stiff cylinders. The dashed line shows the slope of the scattering curve from an ideal rodlike particle.

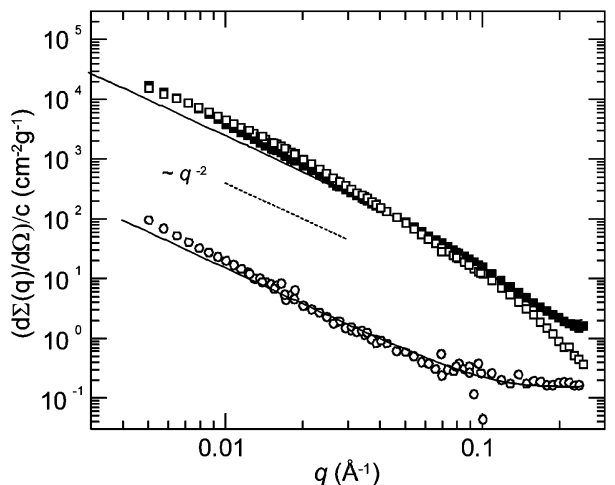

Figure 7. SANS data of PFO in $\mathrm{MCH}-d_{14}$ with concentration of 10 $\mathrm{mg} / \mathrm{mL}$ (solid squares) and $5 \mathrm{mg} / \mathrm{mL}$ (open squares) as well as $10 \mathrm{mg} /$ mL PFO- $d_{34}$ in MCH- $d_{14}$ (open spheres) normalized to concentration. Solid lines are corresponding fits based on the disk model. A dashed line shows the -2 decay for comparison.

Essentially no scattering was detected for side chain deuterated PFO, PFO- $d_{34}$, in toluene- $d_{8}$ (data not shown). The nondeuterated PFO backbone is not detectable due to the size of the monomer. This finding supports our previous conclusions that PFO is dissolved down to the molecular level and that the thickness of the fused-ring system containing only one layer of nuclei is likely not enough to produce sufficient signal to be detected by SANS.

Sheetlike PFO Aggregates in $M C H$. Figure 7 plots SANS data of 10 and $5 \mathrm{mg} / \mathrm{mL}$ PFO as well as $10 \mathrm{mg} / \mathrm{mL}$ PFO- $d_{34}$ in MCH- $d_{14}$. As mentioned above, the decay of $\sim q^{-2}$ seen over large $q$ range for $10 \mathrm{mg} / \mathrm{mL}$ of PFO in $\mathrm{MCH}-d_{14}$ is a strong indication of the existence of 2-dimensional aggregates (sheets or disks) and a striking contrast to the rodlike particles in toluene. A small deviation from -2 behavior at low $q$ can arise from the bending of the obviously not ideally rigid sheets. The scattering patterns of PFO dissolved in $\mathrm{MCH}-d_{14}$ at 5 and 10 $\mathrm{mg} / \mathrm{mL}$ are essentially similar after normalization to concentration. The strong scattering due to the aggregation produces clear signal from side chain deuterated PFO- $d_{34}$ as well and it shows a similar -2 decay as nondeuterated PFO. Unsurprisingly, sidechain deuteration decreases the scattering intensity compared

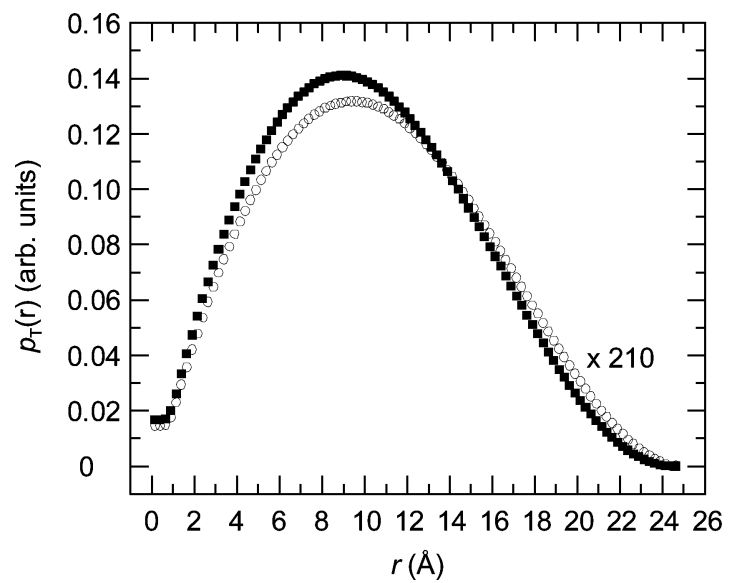

Figure 8. Pair distance distribution functions of thickness, $p_{T}(r)$, obtained from the SANS data of PFO (solid squares) and PFO- $d_{34}$ (open spheres) in MCH- $d_{14}$. The latter is multiplied by the ratio between $I(0)_{\mathrm{PFO}}$ and $I(0)_{\mathrm{PFO}-d_{34}}, 210$. Concentration was $10 \mathrm{mg} / \mathrm{mL}$.

to the completely nondeuterated polymer, and the ratio between $I(0)_{\mathrm{PFO}}$ and $I(0)_{\mathrm{PFO}-d_{34}}$ is 210 .

SANS data of PFO/MCH- $d_{14}$ were analyzed by fitting models of sheetlike particles, polydisperse disks with the mean radius $\langle R\rangle$, standard deviation of normal distributed mean radius, $\sigma$, and thickness $T$. Moreover, the data were treated using a modelindependent IFT approach for sheetlike aggregates, which gave the radius of gyration of thickness, $R_{T, \text { g }}$, the scattering at "zero angle", $I(0)_{T}$, and the pair distance distribution function of thickness, $p_{T}(r)$. These results allowed us to obtain the mass per unit area, $M_{\mathrm{S}}$, and thickness of homogeneous sheet, $T_{\mathrm{S}}$. Figure 8 plots the corresponding pair distribution functions obtained from the SANS data of PFO and PFO- $d_{34}$ in $10 \mathrm{mg} /$ $\mathrm{mL} \mathrm{MCH}-d_{14}$ solutions. The structural parameters thus obtained are compiled in Table 3.

The thickness of the PFO sheets in $\mathrm{MCH}-d_{8}$ is $27.8 \pm 1.0 \AA$ and that of PFO- $d_{34}$ sheets in $\mathrm{MCH}-d_{8}$ practically the same, $28.8 \pm 1.0 \AA$ A . Although the difference between the obtained thicknesses is within the experimental error, the small difference seen in the shape of correlation function can give a hint for the inner structure. The $p_{T}(r)$ of PFO- $d_{34}$ is somewhat broader and shifted toward larger $r$ compared to that of PFO (Figure 8), suggesting that the nondeuterated backbones of the PFO- $d_{34} \mathrm{~S}$ lie close to the outer surface of the sheet, and the side chains are more accumulated inside the sheets. However, a rigorous picture cannot be given at present.

An important question is whether all PFO is forming sheetlike aggregates in the MCH- $d_{14}$ solutions. SANS gives an average picture suggesting dominance of the sheetlike particles for PFO in $\mathrm{MCH}-d_{14}$. The presence of rodlike particles was investigated at large $q$ where scattering from sheetlike structures in $\mathrm{MCH}-$ $d_{14}$ is significantly lower than that from single chains in toluene$d_{8}$ (Figure 4). Incoherent background was also taken into account. However, as shown in Figure 4, the scattering curve of PFO in $\mathrm{MCH}-d_{14}$ is dominated by -2 behavior even at high $q$, indicating dominance of sheets. Altogether, the data show that PFO is dissolved down to the colloidal level in $\mathrm{MCH}-d_{14}$ and forms sheetlike particles. Free rodlike conformations may

Table 2. Parameters Fitted to the SANS Data of PFO in Toluene- $d_{8}{ }^{a}$

\begin{tabular}{cccccc}
\hline material & $L(\AA)$ & $R(\AA)$ & $R_{\mathrm{CS}, \mathrm{g}}(\AA)$ & $R_{\mathrm{c}}(\AA)$ & $M_{\mathrm{L}}(\mathrm{g} / \mathrm{cm}) \times 10^{-15}$ \\
\hline PFO in toluene- $d_{8}, 10 \mathrm{mg} / \mathrm{mL}$ & $710 \pm 50$ & $7.5 \pm 0.2$ & $6.2 \pm 0.1$ & $8.7 \pm 0.3$ & $5.4 \pm 0.2$ \\
PFO in toluene- $d_{8}, 5 \mathrm{mg} / \mathrm{mL}$ & $850 \pm 50$ & $4.3 \pm 0.1$ & $4.3 \pm 0.1$ & $6.1 \pm 0.2$ & $5.8 \pm 0.2$
\end{tabular}

${ }^{a} L$ and $R$ respectively are the length and radius of homogeneous cylinder obtained by the model fitting. $R_{\mathrm{CS}, \mathrm{g}}$ and $R_{\mathrm{c}}$ respectively are the cross-sectional radius of gyration and corresponding radius of homogeneous cross section. $M_{\mathrm{L}}$ is the mass per unit length obtained by the IFT method. 
Table 3. Parameters Fitted to the SANS Data of PFO and PFO- $d_{34}$ in MCH- $d_{14}{ }^{a}$

\begin{tabular}{|c|c|c|c|c|c|c|c|}
\hline material & concn $(\mathrm{mg} / \mathrm{mL})$ & $R_{\mathrm{T}, \mathrm{g}}(\AA)$ & $T_{\mathrm{S}}(\AA)$ & $M_{\mathrm{S}}\left(\mathrm{g} / \mathrm{cm}^{2}\right) \times 10^{8}$ & $\langle R\rangle(\AA)$ & $\sigma^{1 / 2}(\AA)$ & $T(\AA)$ \\
\hline $\mathrm{PFO}$ in $\mathrm{MCH}-d_{14}$ & 5 & $8.6 \pm 0.2$ & $29.8 \pm 1$ & $9.6 \pm 0.1$ & & & \\
\hline $\mathrm{PFO}$ in $\mathrm{MCH}-d_{14}$ & 10 & $8.0 \pm 0.2$ & $27.8 \pm 1$ & $9.7 \pm 0.1$ & $1000 \pm 100$ & $0.35 \pm 0.05$ & $27 \pm 1$ \\
\hline PFO- $d_{34}$ in MCH- $d_{14}$ & 10 & $8.3 \pm 0.2$ & $28.8 \pm 1$ & & $1000 \pm 100$ & $0.35 \pm 0.05$ & $29 \pm 1$ \\
\hline
\end{tabular}

${ }^{a} R_{\mathrm{T}, \mathrm{g}}$ and $T_{\mathrm{S}}$ respectively are the radius of gyration of thickness and the corresponding thickness in homogeneous approximation; $M_{\mathrm{S}}$ is the measured mass per unit area obtained by the IFT method. $\langle R\rangle, \sigma$, and $T$ respectively are the mean radius of disks, standard deviation of disks, and disk thickness.

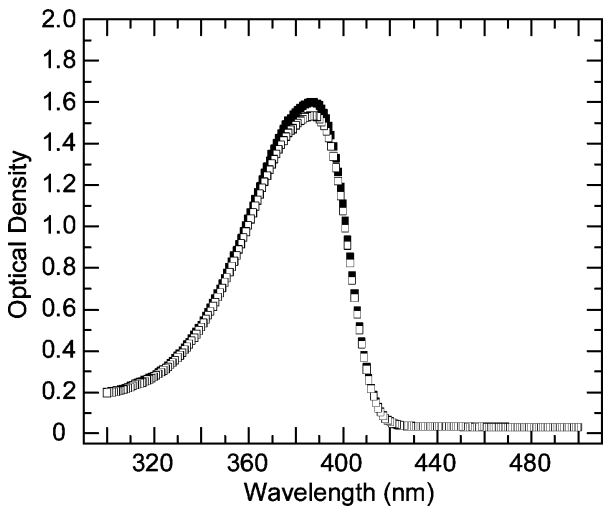

Figure 9. Optical density of PFO in toluene- $d_{8}$ immediately after dissolution at $80{ }^{\circ} \mathrm{C}$ (solid squares) and after cooling at $0{ }^{\circ} \mathrm{C}$ for $4 \mathrm{~h}$ (open squares). Concentration was $10 \mathrm{mg} / \mathrm{mL}$.

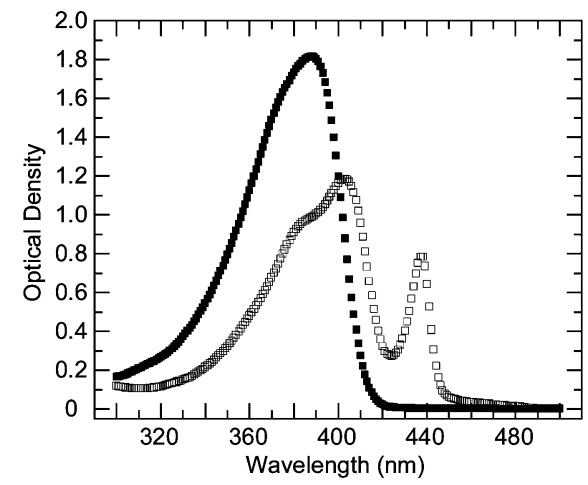

Figure 10. Optical density of PFO in $\mathrm{MCH}-d_{14}$ immediately after dissolution at $80{ }^{\circ} \mathrm{C}$ (solid squares) and after cooling at $0{ }^{\circ} \mathrm{C}$ for $4 \mathrm{~h}$ (open squares). Concentration was $10 \mathrm{mg} / \mathrm{mL}$. In the latter, the ratio of optical densities at $400 \mathrm{~nm}$ over $436 \mathrm{~nm}$ is 0.66 .

be present, but their fraction is smaller. Comparing the intensities at large $q$, it is likely to be much less than $50 \%$.

Conformational Isomers of PFO in Toluene and $\mathrm{MCH}$. Figures 9 and 10 respectively plot the steady-state photoabsorption spectra of PFO in toluene- $d_{8}$ and in MCH- $d_{14}$. The spectra shown in Figure 9 are characteristic for all PFs, and in the case of PFO they are interpreted to arise from the conformational isomer $\mathrm{C}_{\alpha}$ (as defined in ref 26). Figure 10 displays a different situation; an additional absorption peak appearing upon cooling at $425-445 \mathrm{~nm}$ is commensurate with the conformational isomer $\mathrm{C}_{\beta}{ }^{26}$ Essentially similar behavior was observed for $5 \mathrm{mg} / \mathrm{mL}$ solutions.

\section{Discussion}

Considering the results above the following overall notes can be made. The PF2/6 data are in agreement with an assumption of individual PF2/6 chains in solvents; bunches of several (or even two) chains would not pack well. These observations are well in the line with our previous combination of experiment and molecular modeling. ${ }^{12}$ A cross-sectional diameter of the same order of magnitude $(1.8 \pm 0.5 \mathrm{~nm})$ has been observed elsewhere. ${ }^{29}$
The PFO data indicate that in toluene- $d_{8}$ PFO forms rodlike particles regardless of the concentration; both the mass per unit length and the length of particles are essentially the same for 5 and $10 \mathrm{mg} / \mathrm{mL}$ samples. Like for PF2/6 these aggregates are very long ( $>70 \mathrm{~nm})$ and very thin - the radius of cross section of the order of $\sim 0.5-1 \mathrm{~nm}$. A small radius suggests that the observed particles are essentially individual polymer chains and that PFO is dissolved down to the molecular level. This is also the level where similarities and differences of PF2/6 and PFO in toluene- $d_{8}$ should be understood. When the system consists of individual polymer chains, the nanometer scale behavior is essentially determined by the rigidity of the backbone which is the same in both polymers, and hence effectively the same structural parameters are the most plausible result. In contrast, the potential differences in torsion angle due to the slightly different side chains are not observable by SANS.

PF $2 / 6\left(M_{\mathrm{n}}=55 \mathrm{~kg} / \mathrm{mol}, M_{\mathrm{w}}=89 \mathrm{~kg} / \mathrm{mol}\right)$ in toluene ${ }^{29}$ and PFO $\left(M_{\mathrm{n}}=34 \mathrm{~kg} / \mathrm{mol}, M_{\mathrm{w}}=56 \mathrm{~kg} / \mathrm{mol}\right)$ in tetrahydrofuran ${ }^{16}$ have been previously studied using light scattering, and the persistence lengths of $7.0 \pm 0.5$ and $8.5 \pm 1.1 \mathrm{~nm}$, respectively, have been reported. In our work neither polymer shows major signs of flexibility in the Holtzer plots in the range of 4-20 $\mathrm{nm}$ (Supporting Information), and the data fit visually well to the rodlike model. Also, the Holtzer plots (Supporting Information) are in accord with the polydisperse chains with Kuhn segments of the order of $\geq 15-20 \mathrm{~nm}$.

When considering the relation between previous reports ${ }^{16,29}$ and the rodlike model (section II) the following further notes can be made. The $L$ values (Tables 1 and 2 ) do not correspond to the Kuhn segments reported in refs 16 and 29. These values are just parameters which reflect the total length of particles and do not mean their complete stiffness. However, even though the rodlike model underestimates the local bending, it is still illustrative and useful when making a phenomenological distinction between one- and two-dimensional particles. As the apparent flexibility of PFs spreads out of the measured interval of scattering vectors, an application of the semiflexible model would be complicated.

Despite the minimal chemical difference (cf. Figure 1), PF2/6 and PFO behave completely differently in $\mathrm{MCH}-d_{14}$. As indicated by Figure 2, there is only a marginal solution dependence in the structure of PF2/6 which in both solvents shows a thin rod configuration. In contrast, $\mathrm{PFO}$ shows a major difference between rodlike particles in toluene- $d_{8}$ and sheetlike in $\mathrm{MCH}-d_{14}$ (Figure 4). Both polymers in both solvents show only a minimal or negligible concentration effect in the concentration range $5-10 \mathrm{mg} / \mathrm{mL}$.

Like sheets, the Gaussian coils also lead to the -2 power law. It is however not easy to imagine how indisputably stiff PFO can be described as a coil in a poor solvent. We can also exclude the coil conformation from the behavior at large $q$ interval for $5 \mathrm{mg} / \mathrm{mL}$ solutions (Figure 7) where we observe a slope between -3 and -4 . This indicates the presence of distinct interfaces between the aggregate and the solvent, while in the case of coils the slope should not exceed -2 .

It is an issue how the individual chains and sheetlike PFO aggregates in $\mathrm{MCH}$ solutions relate to the crystalline, semi- 
crystalline, liquid crystalline, or amorphous solid-state phases. To clarify this issue, we discuss PFO in terms of both intermolecular crystalline and noncrystalline phases and intramolecular conformational isomers.

In intermolecular (crystallographic) terms, nematic and isotropic phases can occur at high temperatures $\left(\sim 160-300{ }^{\circ} \mathrm{C}\right.$ and above) while it is generally accepted that at the room temperature the solid state is dominated by a stable crystalline $\alpha$-phase, for which an orthorhombic lattice of eight chains with lattice parameters $a=25.6 \AA, b=23.4 \AA$, and $c=3.36 \AA$ (crystallographic $c$-axis being along the polymer backbone) and density $\approx 1.0 \mathrm{~g} / \mathrm{cm}^{3}$ has been proposed..$^{23}$ Another crystalline form, metastable $\alpha^{\prime}$, is comprised of a slightly modified orthogonal lattice along the $b$-axis $(b=23.8 \AA) .{ }^{25}$ Room temperature forms also include an (optically isotropic) amorphous phase and noncrystalline, metastable $\beta$-phase. Although this $\beta$-phase is generally denoted as noncrystalline, it does give rise to sharp Bragg reflections commensurate with lamellar order with a long period of $12.3 \AA .{ }^{24}$ It, however, differs from "real" crystals in a sense that it is rather a mesomorphic phase and requires a presence of absorbed solvent, arising from extended treatment with solvent vapor, for instance. In particular, it has been found as an intermediate stage of transformation from the solvent-induced clathrate structure to the solvent-free crystalline order, $\alpha$-phase. ${ }^{24}$ These phases can further coexist; they are coupled with each other, and it should be understood that the $\beta$-phase is structurally incompatible with $\alpha{ }^{27}$ Yet this picture is an oversimplification. The overall behavior includes moreover a glassy g-phase. ${ }^{55}$

In intramolecular terms, the local inhomogeneities in PFO structure stem from the side-chain conformation (anti and antigauche-gauche), leading to at least three different conformational isomers $\mathrm{C}_{\alpha}, \mathrm{C}_{\beta}$, and $\mathrm{C}_{\gamma},{ }^{26}$ which respectively have torsion angles between the fluorene monomers of $135^{\circ}, 160^{\circ}$, and $150^{\circ 26}$ (or $138^{\circ}, 165^{\circ}$, and $155^{\circ 27}$ ). These variations in the local planarity can have a crossover, but $\mathrm{C}_{\beta}$ consists only of a single isomer. The intramolecular variations then relate to the intermolecular framework, most distinguishably to $\alpha$ - and $\beta$-phases. It is worth noting that the $\beta$-phase is also a generic term that can simply refer to the low-energy absorption/emission state of the chain without any rigorous connection to the true microscopic structural framework.

To dissolve PFO in $\mathrm{MCH}-d_{14}$, heating to $80{ }^{\circ} \mathrm{C}$ was necessary (cf. section II). DSC measurements indicated that PFO studied in the solid state has an order-disorder transition (ODT) at 79.3 $\pm 0.5{ }^{\circ} \mathrm{C}$. It is known that in solid state above this transition PFO exhibits only the $\alpha$-phase. On cooling polymer reverts back to the $\beta$-type conformation although substantial amounts of $\alpha$-phase remain in material. ${ }^{17}$ This represents a kind of solidstate background of our study.

In this work we did not observe an ordered solid-state phase on the nanometer scale. No Bragg peaks or three-dimensional packing were seen. The thickness of PFO rods is always that of the single molecule while the thickness of PFO sheets corresponds to the thickness of $2-3$ polymer chains. In this respect solution and solid state are simply and fundamentally different. Therefore, we have chosen not to mix the solid state and solution phenomena or terminology in our discussion and do not denote observed aggregates by term used for the ordered solid state elsewhere. Yet, the intramolecular framework is an issue, and to probe these distances by SANS is unreachable. In contrast, the optical properties of PFO are intrinsic in origin relating to the planarization of the chromophores and thus the conformation isomers. The planarity of conformational isomers was thus indirectly studied by photoabsorption.

Considering the intermolecular structural states, we obtained markedly different conformations of PFO in the two solvent. However, we do not detect any crystalline $\alpha$ - or $\alpha^{\prime}$-lattices as understood in solid-state literature but long and thin (radius around $1 \mathrm{~nm}$ ) rodlike particles and large sheets with thickness of $\sim 2-3 \mathrm{~nm}$.

Considering intramolecular terms, we note in turn that SANS does not probe local, angstrom scale variations arising from conformational isomers $\mathrm{C}_{\alpha}$ and $\mathrm{C}_{\beta}$ which, however, are clearly present according to the optical density measurements (Figures 9 and 10).

Figure 7 implies that the concentration of sheetlike particles in the $\mathrm{PFO} / \mathrm{MCH}-d_{14}$ system is directly proportional to the overall concentration of PFO. Elsewhere, ${ }^{37}$ it has been reported that the feature at $436 \mathrm{~nm}$ in the optical spectrum of the ultradilute $\mathrm{PFO} / \mathrm{MCH}$ system is also directly proportional to the concentration of PFO. These observations may have an implication of equilibrium nature of the coexistence of rodlike molecules and sheetlike particles.

We finally note that the PFO/MCH- $d_{14}$ samples are metastable, and they undergo slow macrophase separation in a few days after preparation. It is well-known that the $\beta$-phase is metastable, ${ }^{24}$ which supports the idea that PFO/MCH- $d_{14}$ samples are closely related to this phase. The $\beta$-phase is, however, stable enough to enable diffraction studies (see e.g. ref 24). In our work all samples were measured immediately after preparation, and the data of an immediately measured sample were found to be identical to that of a test measurement performed $24 \mathrm{~h}$ after preparation. Therefore, the phase separation does not significantly influence the measurements or alter our interpretation. However, even in the (hypothetical) case where macrophase separation was quicker, SANS would not detect solid PFO as the scattering arises from the contrast between proton- and deuterium-rich domains of nanometer scale. Thus, the sheets observed for PFO/MCH- $d_{14}$ are not crystalline, and they do not locate at the (hypothetical) macrophase-separated particles.

\section{Conclusions}

In summary, we have investigated solvent-induced phase behaviors of PF2/6 and PFO in deuterated toluene and in deuterated $\mathrm{MCH}$ solutions at $20{ }^{\circ} \mathrm{C}$. Essential structural parameters based on fits to cylinders and sheetlike structures and on the IFT method have been given.

Although the chemical difference between PF2/6 and PFO is marginal, the physical difference in the solution structure is distinctive. PF2/6 forms thin $(\sim 1 \mathrm{~nm})$ and long $(>70 \mathrm{~nm})$ elongated particles in toluene and in $\mathrm{MCH}$, those being somewhat thinner in $\mathrm{MCH}$. We interpret these particles as predominantly single polymer chains with the side chains forming more compact corona in the poorer solvent $(\mathrm{MCH})$. PFO shows a rodlike structure in toluene but sheetlike stucture in $\mathrm{MCH}$. The rodlike structure is analogous to that seen for PF2/6 and also composed mainly by individual chains adapting dominantly a conformational isomer $\mathrm{C}_{\alpha}$ as evidenced from photoabsorption spectra. The sheetlike particles (characteristic thickness of $\sim 2-3 \mathrm{~nm}$ ) are suggested to comprise of around two polymer layers. Their exact internal structure cannot be given at present, but the measurement with the side-chain deuterated PFO gives some evidence that the backbones are evenly distributed within the sheets. The optical absorption arising from the samples with sheetlike conformation of PFO in $\mathrm{MCH}$ correlates well to the absorption of the $\beta$-phase in the 
solid state. Hence, the sheets contain conformational isomer $\mathrm{C}_{\beta}$. These sheets can be the initial stage of PFO crystallization, and whether they relate to the $\beta$-phase of the solid-state PFO has been discussed. All observations were found not to depend on the concentration over the range $5-10 \mathrm{mg} / \mathrm{mL}$.

Acknowledgment. This study has been funded by One NorthEast (UK) UIC Nanotechnology grant. M.K. thanks the European Commission for support under the sixth Framework Program through the Key Action-Strengthening the European Research Area, Research Infrastructures (Contract RII3-CT2003-505925) as well as Prof. M. J. Winokur of the University of Wisconsin-Madison for discussions. We also thank S. Weber of the University of Wuppertal for assistance and Prof. X. Ariza of the University of Barcelona for a deuterated monomer. L.A. acknowledges support of the Széchenyi István Scholarship Foundation.

Supporting Information Available: Holtzer plots of the SANS data of PF2/6 and PFO in toluene- $d_{8}$ as well as the details of IFT analysis and a model of stiff interacting cylinders. This material is available free of charge via the Internet at http://pubs.acs.org.

\section{References and Notes}

(1) Grosberg, A. Y.; Khokhlov, A. R. Statistical Physics of Macromolecules; American Institute of Physics: Woodbury, NY, 1994.

(2) Wegner, G. Macromol. Chem. Phys. 2003, 204, 347-357.

(3) Winokur, M. J. Structure Studies of pi- and sigma-Conjugated Polymers. In Handbook of Conducting Polymers; Skotheim, T. A., Elsenbaumer, R. L., Reynolds, J. R., Eds.; Taylor \& Francis: London, 2006.

(4) Knaapila, M.; Stepanyan, R.; Lyons, B. P.; Torkkeli, M.; Monkman, A. P. Adv. Funct. Mater. 2006, 16, 599-609.

(5) Neher, D. Macromol. Rapid Commun. 2001, 22, 1365-1385.

(6) Scherf, U.; List, E. J. W. Adv. Mater. 2002, 14, 477-487.

(7) Lieser, G.; Oda, M.; Miteva, T.; Meisel, A.; Nothofer, H.-G.; Scherf, U.; Neher, D. Macromolecules 2000, 33, 4490-4495.

(8) Knaapila, M.; Lyons, B. P.; Kisko, K.; Foreman, J. P.; Vainio, U.; Mihaylova, M.; Seeck, O. H.; Pålsson, L.-O.; Serimaa, R.; Torkkeli, M.; Monkman, A. P. J. Phys. Chem. B 2003, 107, 12425-12430.

(9) Tanto, B.; Guha, S.; Martin, C. M.; Scherf, U.; Winokur, M. J. Macromolecules 2004, 37, 9438-9448.

(10) Knaapila, M.; Kisko, K.; Lyons, B. P.; Stepanyan, R.; Foreman, J. P.; Seeck, O. H.; Vainio, U.; Pålsson, L.-O.; Serimaa, R.; Torkkeli, M.; Monkman, A. P. J. Phys. Chem. B 2004, 108, 10711-10720.

(11) Knaapila, M.; Stepanyan, R.; Lyons, B. P.; Torkkeli, M.; Hase, T. P. A.; Serimaa, R.; Güntner, R.; Seeck, O. H.; Scherf, U.; Monkman, A. P. Macromolecules 2005, 38, 2744-2753.

(12) Knaapila, M.; Stepanyan, R.; Torkkeli, M.; Lyons, B. P.; Ikonen, T. P.; Almásy, L.; Foreman, J. P.; Serimaa, R.; Güntner, R.; Scherf, U.; Monkman, A. P. Phys. Rev. E 2005, 71, 041802.

(13) Knaapila, M.; Lyons, B. P.; Hase, T. P. A.; Pearson, C.; Petty, M. C.; Bouchenoire, L.; Thompson, P.; Serimaa, R.; Torkkeli, M.; Monkman, A. P. Adv. Funct. Mater. 2005, 15, 1517-1522.

(14) Chi, C.; Lieser, G.; Enkelmann, V.; Wegner, G. Macromol. Chem. Phys. 2005, 206, 1597-1609.

(15) Marcon, V.; van der Vegt, N.; Wegner, G.; Raos, G. J. Phys. Chem. B 2006, 110, 5253-5261.

(16) Grell, M.; Bradley, D. D. C.; Long, X.; Chamberlain, T.; Inbasekaran, M.; Woo, E. P.; Soliman, M. Acta Polym. 1998, 79, 439-444.

(17) Grell, M.; Bradley, D. D. C.; Ungar, G.; Hill, J.; Whitehead, K. S. Macromolecules 1999, 32, 5810-5817.

(18) Ariu, M.; Lidzey, D. G.; Bradley, D. D. C. Synth. Met. 2000, 111$112,607-610$.

(19) Kawana, S.; Durrell, M.; Lu, J.; MacDonald, J. E.; Grell, M.; Bradley, D. D. C.; Jukes, P. C.; Jones, R. A. L.; Bennett, S. L. Polymer 2002, 43, 1907-1913.
(20) Winokur, M. J.; Slinker, J.; Huber, D. L. Phys. Rev. B 2003, 67, 184106.

(21) Misaki, M.; Ueda, Y.; Nagamatsu, S.; Yoshida, Y.; Tanigaki, N.; Yase, K. Macromolecules 2004, 37, 6926-6931.

(22) Rothe, C.; King, S. M.; Dias, F.; Monkman, A. P. Phys. Rev. B 2004 70, 195213.

(23) Chen, S. H.; Chou, H. L.; Su, A. C.; Chen, S. A. Macromolecules 2004, 37, 6833-6838.

(24) Chen, S. H.; Su, A. C.; Chen, S. A. J. Phys. Chem. B 2005, 109, 10067-10072.

(25) Chen, S. H.; Su, A. C.; Su, C. H.; Chen, S. A. Macromolecules 2005, $38,379-385$

(26) Chunwaschirasiri, W.; Tanto, B.; Huber, D. L.; Winokur, M. J. Phys. Rev. Lett. 2005, 94, 107402.

(27) Arif, M.; Volz, C.; Guha, S. Phys. Rev. Lett. 2006, 96, 025503.

(28) Chen, S. H.; Su, A. C.; Su, C. H.; Chen, S. A. J. Phys. Chem. B 2006, 110, 4007-4013.

(29) Fytas, G.; Nothofer, H. G.; Scherf, U.; Vlassopoulos, D.; Meier, G. Macromolecules 2002, 35, 481-488.

(30) Wu, L.; Sato, T.; Tang, H.-Z.; Fujiki, M. Macromolecules 2004, 37, 6183-6188.

(31) Banach, M. J.; Friend, R. H.; Sirringhaus, H. Macromolecules 2004, $37,6079-6085$.

(32) Larez-V, C.; Crescenzi, V.; Ciferri, A. Macromolecules 1995, 28 , $5280-5284$.

(33) Bright, J. N.; Williams, D. R. M. Europhys. Lett. 1999, 45, 321-326.

(34) Limbach, H. J.; Holm, C.; Kremer, K. Macromol. Chem. Phys. 2005 , 206, 77-82.

(35) Ou-Yang, W. C.; Chang, C.-S.; Chen, H.-L.; Tsao, C.-S.; Peng, K.Y.; Chen, S.-A.; Han, C. C. Phys. Rev. E 2005, 72, 031802.

(36) Blondin, P.; Bouchard, J.; Beaupré, S.; Belletête, M.; Durocher, G.; Leclerc, M. Macromolecules 2000, 33, 5874-5879.

(37) Dias, F. B.; Macanita, A. L.; Burrows, H. D.; Morgado, J.; Monkman, A. P., unpublished results.

(38) Guilleaume, B.; Blaul, J.; Wittemann, M.; Rehahn, M.; Ballauff, M. J. Phys.: Condens. Matter 2000, 12, A245-A251

(39) Guilleaume, B.; Blaul, J.; Ballauff, M.; Wittemann, M.; Rehahn, M.; Goerigk, G. Eur. Phys. J. E 2002, 8, 299-309.

(40) Hickl, P.; Ballauff, M.; Scherf, U.; Müllen, K.; Lindner, P. Macromolecules 1997, 30, 273-279.

(41) Wang, D.; Lal, J.; Moses, D.; Bazan, G. C.; Heeger, A. J. Chem. Phys. Lett. 2001, 348, 411-415.

(42) Zaroslov, Yu. D.; Gordeliy, V. I.; Kuklin, A. I.; Islamov, A. H.; Philippova, O. E.; Khokhlov, A. R.; Wegner, G. Macromolecules 2002, 35, 4466-4471.

(43) Chen, S. H.; Su, A. C.; Chang, C. S.; Chen, H. L.; Ho, D. L.; Tsao, C. S.; Peng, K. Y.; Chen, S. A. Langmuir 2004, 20, 8909-8915.

(44) Dias, F. B.; Knaapila, M.; Monkman, A. P.; Burrows, H. D. Macromolecules 2006, 39, 1598-1606.

(45) The term "rod" is used in a phenomenological fashion just to make a distinction between "rods" and "sheets". This term does not mean an overall rod shape of polymer chains.

(46) Galbrecht, F.; Yang, X. H.; Nehls, B. S.; Neher, D.; Farrell, T.; Scherf, U. Chem. Commun. 2005, 18, 2378-2380.

(47) Rosta, L. Appl. Phys. A 2002, 74, S52-S54.

(48) Stuhrmann, H. B.; Burkhardt, N.; Dietrich, G.; Jünemann, R.; Meerwinck, W.; Schmitt, M.; Wadzack, J.; Willumeit, R.; Zhao, J.; Nierhaus, K. H. Nucl. Instrum. Methods A 1995, A356, 124-132.

(49) Wignall, G. D.; Bates, F. S. J. Appl. Crystallogr. 1987, 20, 28-40.

(50) Pedersen, J. S.; Schurtenberger, P. Macromolecules 1996, 29, 76027612.

(51) Pedersen, J. S. Adv. Colloid Interface Sci. 1997, 70, 171-210.

(52) Jerke, G.; Pedersen, J. S.; Egelhaaf, S. U.; Schurtenberger, P. Phys. Rev. E 1997, 56, 5772-5788

(53) Glatter, O. J. Appl. Crystallogr. 1977, 10, 415-421.

(54) Note that PF2/6 does not form genuine crystals but quasicrystals and paracrystals, and lattice parameters have been used here just to make a rough phenomenological comparison between the structures in solvent and solid state.

(55) Becker, K.; Lupton, J. M. J. Am. Chem. Soc. 2005, 127, 7306-7307. MA060886C 